\title{
Erratum to: Diversity oriented synthesis for novel anti-malarials
}

\author{
Chandramohan Bathula $^{1} \cdot$ Shailja Singh $^{2} \cdot$ Subhabrata Sen $^{1}$
}

Published online: 18 July 2015

(C) Springer Science+Business Media Dordrecht 2015

\section{Erratum to: Syst Synth Biol \\ DOI 10.1007/s11693-015-9171-0}

In the original publication of the article, the first two sentences of the abstract were wrongly published. The correct abstract is given below.

Abstract Malaria is perhaps one of the most lethal infectious diseases that have exterminated nearly half a billion population of the world. Growing resistance of malarial strains to artemisinin therapy, the only available viable cure for malaria, has made the situation grimmer. Hence, identifying new cure for malaria is the need of the hour. Our commentary discusses the advent and contribution of diversity oriented synthesis (DOS) in the drug discovery efforts towards developing cure for malaria. DOS based on chemical genetics focusses on design and synthesis of molecular libraries which covers large tracts of biologically relevant chemical space. Herein, we will discuss the applications, advantages, disadvantages and future directions of DOS with respect to malaria.

The online version of the original article can be found under doi:10.1007/s11693-015-9171-0.

Subhabrata Sen

subhabrata.sen@snu.edu.in

1 Department of Chemistry, School of Natural Science, Shiv

Nadar University, Village Chithera, Tehsil Dadri,

District Gautam Buddha Nagar 201314, UP, India

2 Department of Life Science, School of Natural Science, Shiv

Nadar University, Village Chithera, Tehsil Dadri,

District Gautam Buddha Nagar 201314, UP, India 